\title{
Video Article \\ Rapid Neuronal Differentiation of Induced Pluripotent Stem Cells for Measuring Network Activity on Micro-electrode Arrays
}

\author{
Monica Frega ${ }^{\star^{1,2}}$, Sebastianus H. C. van Geste ${ }^{*^{3}}$, Katrin Linda ${ }^{2,3}$, Jori van der Raadt ${ }^{3}$, Jason Keller ${ }^{1,2}$, Jon-Ruben Van Rhijn ${ }^{1,2}$, Dirk Schubert ${ }^{1,2}$, \\ Cornelis A. Albers ${ }^{2,3,4}$, Nael Nadif Kasri ${ }^{1,2,3}$ \\ ${ }^{1}$ Department of Cognitive Neurosciences, Radboudumc \\ ${ }^{2}$ Donders Institute for Brain, Cognition and Behaviour, Radboud University \\ ${ }^{3}$ Department of Human Genetics, Radboudumc \\ ${ }^{4}$ Department of Molecular Developmental Biology, Radboud University \\ *These authors contributed equally
}

Correspondence to: Cornelis A. Albers at kees.albers@radboudumc.nl, Nael Nadif Kasri at n.nadif@donders.ru.nl

URL: https://www.jove.com/video/54900

DOI: doi: $10.3791 / 54900$

Keywords: Developmental Biology, Issue 119, induced pluripotent stem cells, neuronal differentiation, micro-electrode arrays, lentiviral transduction, astrocyte isolation, neuronal network

Date Published: 1/8/2017

Citation: Frega, M., van Gestel, S.H., Linda, K., van der Raadt, J., Keller, J., Van Rhijn, J.R., Schubert, D., Albers, C.A., Nadif Kasri, N. Rapid Neuronal Differentiation of Induced Pluripotent Stem Cells for Measuring Network Activity on Micro-electrode Arrays. J. Vis. Exp. (119), e54900, doi:10.3791/54900 (2017).

\section{Abstract}

Neurons derived from human induced Pluripotent Stem Cells (hiPSCs) provide a promising new tool for studying neurological disorders. In the past decade, many protocols for differentiating hiPSCs into neurons have been developed. However, these protocols are often slow with high variability, low reproducibility, and low efficiency. In addition, the neurons obtained with these protocols are often immature and lack adequate functional activity both at the single-cell and network levels unless the neurons are cultured for several months. Partially due to these limitations, the functional properties of hiPSC-derived neuronal networks are still not well characterized. Here, we adapt a recently published protocol that describes production of human neurons from hiPSCs by forced expression of the transcription factor neurogenin- $2^{12}$. This protocol is rapid (yielding mature neurons within 3 weeks) and efficient, with nearly $100 \%$ conversion efficiency of transduced cells (>95\% of DAPI-positive cells are MAP2 positive). Furthermore, the protocol yields a homogeneous population of excitatory neurons that would allow the investigation of celltype specific contributions to neurological disorders. We modified the original protocol by generating stably transduced hiPSC cells, giving us explicit control over the total number of neurons. These cells are then used to generate hiPSC-derived neuronal networks on micro-electrode arrays. In this way, the spontaneous electrophysiological activity of hiPSC-derived neuronal networks can be measured and characterized, while retaining interexperimental consistency in terms of cell density. The presented protocol is broadly applicable, especially for mechanistic and pharmacological studies on human neuronal networks.

\section{Video Link}

The video component of this article can be found at https://www.jove.com/video/54900/

\section{Introduction}

The development of human induced Pluripotent Stem Cells (hiPSCs) differentiation protocols to generate human neurons in vitro has provided a powerful new tool for studying neurological disorders. Until recently, the study of these disorders was severely hampered by the lack of model systems using human neurons. Although rodents can be used to study neurological disorders, the results of such studies cannot be translated easily to humans ${ }^{1}$. Given these limitations, hiPSC-derived neurons are a promising alternative model that can be used to elucidate molecular mechanisms underlying neurological disorders and for in vitro drug screening.

In the past decade, several protocols to convert hiPSCs into neurons have been developed ${ }^{2-8}$. However, these protocols are still limited in many ways. First, the protocols are often time-consuming: generating neurons with adequate maturation (i.e. synapse formation) and functional activity requires months of culturing procedures, which renders large-scale studies difficult ${ }^{9}$. In addition, hiPSC-to-neuron conversion efficiency is low. Protocols often yield a heterogeneous population of neurons, and thus do not allow studies of specific subsets of neuronal cells. Moreover, the protocols are not reproducible, yielding different results for different iPSC lines ${ }^{10,11}$. Lastly, the maturation stage and functional properties of the resulting neurons are also variable ${ }^{10}$.

To address these problems, Zhang et al. $(2013)^{12}$ developed a protocol that rapidly and reproducibly generates human neurons from hiPSCs by overexpressing the transcription factor neurogenin-2. As reported by the authors, differentiation occurs relatively quickly (only $2-3$ weeks after inducing expression of neurogenin-2), the protocol is reproducible (neuronal properties are independent of the starting hiPSC line), and the hiPSC-to-neuron conversion is highly efficient (nearly $100 \%$ ). The population of neurons generated with their protocol is homogeneous 
(resembling upper-layer cortical neurons), allowing the investigation of cell-type specific contributions to neuronal disorders. Furthermore, their hiPSC-derived neurons exhibited mature properties (e.g., the capability to form synapses and robust functional activity) after only $20 \mathrm{~d}$.

Characterizing the electrophysiological properties of hiPSC-derived neurons at the network level is an important prerequisite before hiPSC technology can be exploited for the study of human diseases. For this reason, many research groups have recently begun to investigate stemcell-derived neurons at the network level using micro-electrode array (MEA) devices (Multichannel Systems, Reutlingen, Germany) ${ }^{13-16}$. The electrodes of a MEA are embedded in a substrate on which neuronal cells can be cultured. MEAs can be used to explore the electrophysiological properties of neuronal networks and the in vitro development of their activity. Currently, MEAs are used only in combination with differentiation protocols that take several months to yield mature networks. Hence, combining MEAs with a rapid differentiation protocol should facilitate the use of this technology in large-scale studies of neurological disorders.

Here, we present a modification of the Zhang et al. (2013) ${ }^{12}$ protocol and adapt it for use on MEAs. In particular, rather than relying on an acute lentiviral transduction, we instead created hiPSC lines stably expressing rtTA/Ngn2 before inducing differentiation. We did this primarily to have reproducible control over the neuronal cell density, since the neuronal cell density is critical for neuronal network formation, and for good contact between the neurons and the electrodes of the MEA ${ }^{17,18}$. Although the Zhang et al. protocol is very efficient with respect to conversion of transduced hiPSCs, it is inherently variable with respect to the final yield of neurons from the number of hiPSCs plated initially (see Figure $2 \mathrm{E}$ in Zhang et al. $)^{12}$. With a stable line, we eliminate many issues causing variability, such as lentiviral toxicity and infection efficiency. We then optimized the parameters that reliably produce hiPSC-derived neuronal networks on MEAs, obtaining network maturation (e.g., synchronous events involving a majority of the channels) by the third week. This rapid and reliable protocol should enable direct comparisons between neurons derived from different (i.e. patient-specific) hiPSC lines as well as provide robust consistency for pharmacological studies.

\section{Protocol}

All experiments on animals were carried out in accordance with the approved animal care and use guidelines of the Animal Care Committee, Radboud University Medical Centre, the Netherlands, (RU-DEC-2011-021, protocol number: 77073).

\section{Glia Cell Isolation and Culture}

NOTE: The protocol presented here is based on the work of McCarthy and de Vellis ${ }^{19}$, and a very similar detailed protocol for mouse astrocytes is available $^{20}$. To generate primary cultures of cortical astrocytes from embryonic (E18) rat brains, a pregnant rat needs to be sacrificed, the embryos need to be harvested from the uterus, and the brains need to be isolated from the embryos. To fill a T75 flask, the cortices from 2 embryonic brains need to be combined. As an alternative, commercially available purified and frozen astrocytes can be purchased.

1. Prepare the T75 culture flask

1. Dilute Poly-D-Lysine (PDL) in sterile, ultrapure water to a final concentration of $10 \mu \mathrm{g} / \mathrm{mL}$. Add $5 \mathrm{~mL}$ of the diluted PDL to the T75 culture flask. Swish around gently to wet the entire growth surface. Place the flask in a humidified $37^{\circ} \mathrm{C}$ incubator for $3 \mathrm{~h}$.

2. Aspirate the PDL from the flask. Rinse the flask $3 x$ with $5 \mathrm{~mL}$ sterile water to remove unbound PDL. Aspirate the water completely. Leave the flask to dry in a laminar flow hood or used immediately.

\section{Dissection of the cortices}

1. Prepare $50 \mathrm{~mL}$ dissection medium: Lebovitz's L-15 medium with $2 \%$ (v/v) B-27 supplement. Keep on ice.

2. Anesthetize the rat deeply with isoflurane in an induction chamber (small Plexiglas box) until respiration ceases ( $\sim 5$ - 8 min). Remove rat from the induction chamber and immediately euthanized by cervical dislocation.

3. Spray the abdomen of the rat with $70 \% \mathrm{EtOH}$ and wipe away the excess. Expose and remove the uterus from the dam via Caesarean section using a pair of scissors ${ }^{21}$.

4. Cut individual embryos from their amniotic sacs with scissors, transfer to a sterile Petri dish filled with cold dissection medium, and keep on ice.

5. Transfer embryos again to a new, sterile $6 \mathrm{~cm}$ Petri dish filled with cold dissection medium. Extract brains from the embryos under a stereo microscope. To expose the brain, gently peel away the skin and skull using forceps. Gently scoop out the entire brain and transfer to a $35 \mathrm{~mm}$ Petri dish with fresh, cold dissection medium.

NOTE: Whole brains dissected from embryos can be stored in dissection medium on ice for many hours without losing cellular viability.

6. Separate the two hemispheres of each brain by cutting through the midline with fine-tipped spring scissors or a scalpel. Carefully strip off the meninges with straight fine-tipped forceps.

NOTE: It is very important to remove the meninges completely. This prevents fibroblast contamination of the astrocyte culture. Fibroblasts are rapidly dividing cells and will eventually displace the other cells.

7. Remove the midbrain/striatum and the olfactory bulb with spring scissors or a scalpel. Also make sure to remove the hippocampus (C-shaped structure that is perimedian and caudal with respect to the cortex) with spring scissors or a scalpel. Collect the cortical hemispheres in a $15 \mathrm{~mL}$ centrifuge tube filled with $5 \mathrm{~mL}$ dissection medium. Keep on ice.

3. Dissociation of the cortices

1. Prepare $2 \mathrm{~mL} \mathrm{Ca}{ }^{2+} / \mathrm{Mg}^{2+}$-free Hank's Balanced Salt Solution (HBSS) with $0.25 \%$ trypsin (dissociation medium). Prepare $50 \mathrm{~mL}$ highglucose Dulbecco's Modified Eagle's Medium (DMEM) with 15\% (v/v) Fetal Bovine Serum (FBS) and 1\% (v/v) penicillin/streptomycin (culture medium) and filter sterilize.

2. Let the tissue settle to the bottom of the centrifuge tube. Carefully aspirate as much of the dissection medium as possible from above the tissue. Wash the tissue with $5 \mathrm{~mL} \mathrm{Ca}{ }^{2+} / \mathrm{Mg}^{2+}$-free HBSS (without trypsin) and allow the tissue to settle at the bottom of the tube.

3. Carefully aspirate the HBSS. Add $2 \mathrm{~mL}$ dissociation medium and flick the tube gently to mix the enzyme around the tissue. Incubate in a $37{ }^{\circ} \mathrm{C}$ water bath for 5-10 min. Flick the tube a few times during incubation to agitate the tissue.

4. Immediately triturate the tissue using a $1,000 \mu \mathrm{L}$ pipette tip. Set the pipetter volume to about $800 \mu \mathrm{L}$. Aspirate the pieces and eject forcefully onto the side of the tube, directly above the fluid line. However, try to minimize bubbles or foaming. Repeat until the tissue 
is sufficiently dissociated, about $15-20 x$. Add $8 \mathrm{~mL}$ culture medium to inactivate the trypsin. Gently mix by inverting the tube several times.

5. Pass the cell suspension through a $70 \mu \mathrm{m}$ cell strainer placed on top of a $50 \mathrm{~mL}$ centrifuge tube. Rinse the $15 \mathrm{~mL}$ tube with culture medium and filter the medium through the cell strainer to collect the medium in the $50 \mathrm{~mL}$ tube with the cell suspension. Rinse the cell strainer a few times with culture medium. After rinsing, the final volume should be about $20-25 \mathrm{~mL}$.

6. Pellet the cells at $200 \mathrm{xg}$ for $10 \mathrm{~min}$. Carefully aspirate as much medium as possible, without touching the cell pellet. Resuspend the cells in $1 \mathrm{~mL}$ culture medium using a $1000 \mu \mathrm{L}$ pipette. Add $11 \mathrm{~mL}$ prewarmed culture medium and mix gently (to prevent bubbles) using a $10 \mathrm{~mL}$ pipette.

7. Rinse the PDL-coated T75 flask once with $5 \mathrm{~mL}$ culture medium. Aspirate the medium and transfer the cell suspension to the flask. All cells are in the suspension are plated, and we find it generally unnecessary to count them, since astrocytes cannot be differentiated from other cells in the suspension. Place the flask into a humidified $37^{\circ} \mathrm{C}$ incubator with an atmosphere of $5 \% \mathrm{CO}_{2}$ for two days.

\section{Expansion and maintenance of the astrocytes}

1. Replace the entire medium for the first time $2 \mathrm{~d}$ after initial plating. Replace the entire medium afterwards every $3 \mathrm{~d}$. Always prewarm the fresh medium to $37^{\circ} \mathrm{C}$ before adding to the cells.

NOTE: The astrocytes require $7-10 \mathrm{~d}$ to reach approximately $90 \%$ confluency (the astrocytes appear as a densely packed tessellated monolayer, with microglia and oligodendrocytes lying on top and intermixed).

2. When the astrocytes reach approximately $90 \%$ confluency, shake the flask to remove the contaminating glial cells:

1. Remove the flask from the incubator and tighten the cap (phenolic) or cover the port (filtered). To remove microglia, shake the flask on an orbital platform at $180 \mathrm{rpm}$ for $1 \mathrm{~h}$. Aspirate the medium. Rinse once with $5 \mathrm{~mL}$ pre-warmed culture medium, aspirate and replace with $12 \mathrm{~mL}$ culture medium.

2. To remove the oligodendrocytes, return the flask to the orbital platform and shake at $250 \mathrm{rpm}, 37^{\circ} \mathrm{C}$ for a minimum of $7 \mathrm{~h}$, but preferably $\mathrm{O} / \mathrm{N}$.

3. Aspirate the medium. Rinse once with $5 \mathrm{~mL}$ pre-warmed culture medium, aspirate and replace with $12 \mathrm{~mL}$ culture medium. Return the flask to the incubator.

4. When $100 \%$ confluent, split the astrocytes using standard procedures at a ratio of $1: 3$ to $1: 2$ with $0.05 \%$ trypsinethylenediaminetetraacetic acid (EDTA). A T75 flask at $100 \%$ confluency will typically yield about $4.0 \times 10^{6}$ cells in total. Under this schedule, the cultures can typically be split once per week.

NOTE: When the astrocytes reach confluency, they can be harvested and used for hiPSC differentiation as described below in protocol step 3.4. The astrocytes can be split at least once without a noticeable loss of viability. They can be maintained for up to 2 months in culture. From experience, primary embryonic day 18 rat astrocytes progressively become terminally differentiated and/or lose viability after repeated splitting. Although it is possible to freeze the astrocytes for future use, we prefer to isolate the astrocytes from fresh embryonic brains when required.

\section{Generation of rtTA/Ngn2-positive hiPSCs}

NOTE: The hiPSCs used for our experiments were generated in-house by lentiviral transduction of human fibroblasts with the reprogramming factors $C M Y C$, SOX2, OCT4 and KLF4.

NOTE: For the generation of $r$ TA/Ngn2-positive hiPSCs, lentiviral vectors are used to stably integrate the transgenes into the genome of the hiPSCs. The protocol for the production of the lentivirus has been published previously ${ }^{22}$. The details of the lentiviral packaging vectors that are used to produce the rtTA and Ngn2 lentivirus particles are provided in the Table of Materials/Equipment. The transfer vector used for the rtTA lentivirus is pLVX-EF1a-(Tet-On-Advanced)-IRES-G418(R); i.e. this vector encodes a Tet-On Advanced transactivator under control of a constitutive EF1 $\alpha$ promoter and confers resistance to the antibiotic G418. The transfer vector used for the Ngn2 lentivirus is pLVX-(TRE-thight)-(MOUSE)Ngn2PGK-Puromycin(R); i.e. this vector encodes the gene for murine neurogenin-2 under control of a Tet-controlled promoter and the puromycin resistance gene under control of a constitutive PGK promoter. Hence, by using these two transfer vectors, an hiPSC line can be created for which the expression of murine neurogenin-2 can be induced by supplementing the medium with doxycycline. For the transduction of the hiPSCs, the supernatant with the lentivirus particles is used (referred to as 'lentivirus suspension' in the remainder of the text), i.e. without concentrating the particles using ultracentrifugation.

1. Plate the hiPSCs (day 1)

NOTE: The volumes that are mentioned in this protocol assume that the hiPSCs are cultured in a 6 well plate and that the cells of one well are harvested. In addition, it is assumed that the cells are plated subsequently in 12 wells of a 12 well plate.

1. Prepare $10 \mathrm{~mL}$ cold DMEM/F12 with $1 \%(\mathrm{v} / \mathrm{v})$ Basement Membrane Matrix (BMM) to obtain diluted BMM. Add $800 \mu \mathrm{L}$ diluted BMM per well of a 12 well plate. Incubate for at least $1 \mathrm{~h}$ in a humidified $37^{\circ} \mathrm{C}$ incubator with an atmosphere of $5 \% \mathrm{CO}_{2}$. Before usage, incubate the plate for $1 \mathrm{~h}$ at RT.

2. Warm $15 \mathrm{~mL}$ Essential 8 (E8) medium with $1 \%(\mathrm{v} / \mathrm{v})$ penicillin/streptomycin, $9 \mathrm{~mL}$ DMEM/F12 and $1 \mathrm{~mL}$ cell detachment solution (CDS) to room temperature. Supplement the E8 medium with Rho-associated protein kinase (ROCK) inhibitor.

3. Aspirate the spent medium of the hiPSCs and add $1 \mathrm{~mL}$ CDS to the hiPSCs. Incubate $3-5$ min in a humidified $37^{\circ} \mathrm{C}$ incubator with an atmosphere of $5 \% \mathrm{CO}_{2}$. Check under the microscope whether the cells are detaching from one another.

4. Add $2 \mathrm{~mL}$ DMEM/F12 in the well, gently suspend the cells with a $1,000 \mu \mathrm{L}$ pipette and transfer the cells to a $15 \mathrm{~mL}$ tube. Add $7 \mathrm{~mL}$ DMEM/F12 to the cell suspension. Spin the cells at $200 \times \mathrm{g}$ for $5 \mathrm{~min}$.

5. Aspirate the supernatant and add $2 \mathrm{~mL}$ of the prepared E8 medium. Obtain a cell suspension in which the hiPSCs are dissociated (do not form cell clumps) by putting the tip of a $1,000 \mu \mathrm{L}$ pipette against the side of the $15 \mathrm{~mL}$ tube and resuspending the cells gently. Check under the microscope whether the cells are dissociated.

6. Determine the number of cells (cells $/ \mathrm{mL}$ ) using a hemocytometer chamber. NOTE: A 6 well plate well at $80-90 \%$ confluency will typically yield $3.0-4.0 \times 10^{6}$ cells in total. 
7. Aspirate the diluted BMM from the wells of the 12 well plate. Dilute the cells to obtain a cell suspension of $3.0 \times 10^{4}$ cells $/ \mathrm{mL}$. Plate 1 $\mathrm{mL}$ of the cell suspension per well of the 12 well plate. Place the 12 well plate $\mathrm{O} / \mathrm{N}$ in a humidified $37^{\circ} \mathrm{C}$ incubator with an atmosphere of $5 \% \mathrm{CO}_{2}$.

2. Transduce the iPS cells with rtTA and Ngn2 lentivirus (day 2)

1. Warm $12 \mathrm{~mL}$ E8 medium with $1 \%(\mathrm{v} / \mathrm{v})$ penicillin/streptomycin to room temperature. Supplement the E8 medium with ROCK inhibitor and polybrene to a final concentration of $8 \mu \mathrm{g} / \mathrm{mL}$ to the E8 medium.

2. Thaw the aliquots with lentivirus suspension. Add polybrene to a final concentration of $8 \mu \mathrm{g} / \mathrm{mL}$ to the lentivirus suspension. Aspirate the spent medium and add $1 \mathrm{~mL}$ of the prepared E8 medium to each well.

3. Perform the transduction with different amounts of the rtTA- and Ngn2-lentivirus suspensions. For example, transduce the hiPSCs by adding $100 \mu \mathrm{L}$ of both the rtTA-lentivirus and Ngn2-lentivirus suspension to one well of the 12 well plate. For the other wells, use 200 $\mu \mathrm{L}, 300 \mu \mathrm{L}, 400 \mu \mathrm{L}$ and $500 \mu \mathrm{L}$ lentivirus suspension instead of $100 \mu \mathrm{L}$. The hiPSCs of two wells of the 12 well plate should not be transduced; they will serve as controls during the selection.

NOTE: The transductions are preferably performed in duplicate, so that the transduction efficiency can be estimated more accurately after the start of the selection (see protocol step 2.2.4). The amount of lentivirus suspension that is required to efficiently transduce the majority of the hiPSCs depends on the titer of the lentivirus suspension and the hiPSC line that is used. In this study, we usually use $100-500 \mu \mathrm{L}$ of lentivirus suspension to transduce the hiPSCs.

4. Place the 12 well plate in a humidified $37^{\circ} \mathrm{C}$ incubator with an atmosphere of $5 \% \mathrm{CO}_{2}$ for $6 \mathrm{~h}$. Before the end of the $6 \mathrm{~h}$ incubation period, warm $12 \mathrm{~mL}$ E8 medium with $1 \%$ (v/v) penicillin/streptomycin and $12 \mathrm{~mL}$ Dulbecco's Phosphate-buffered Saline (DPBS) to RT. Supplement the E8 medium with ROCK inhibitor.

5. Aspirate the spent E8 medium. Wash each well with $1 \mathrm{~mL}$ DPBS. Add $1 \mathrm{~mL}$ of the prepared E8 medium to each well. Place the $12 \mathrm{well}$ plate $\mathrm{O} / \mathrm{N}$ in a humidified $37^{\circ} \mathrm{C}$ incubator with an atmosphere of $5 \% \mathrm{CO}_{2}$.

3. Refresh the E8 medium (day 3)

1. Warm $12 \mathrm{~mL}$ E8 medium with $1 \%(\mathrm{v} / \mathrm{v})$ penicillin/streptomycin to RT. Aspirate the spent medium from the wells of the $12 \mathrm{well}$ plate and add $1 \mathrm{~mL}$ of the prepared $\mathrm{E} 8$ medium to each well. Place the 12 well plate $\mathrm{O} / \mathrm{N}$ in a humidified $37^{\circ} \mathrm{C}$ incubator with an atmosphere of $5 \% \mathrm{CO}_{2}$

4. Perform selection with puromycin and G418 (day 4 - 8)

NOTE: Depending on the cell division rate of the hiPSC line and the efficiency of the lentiviral transduction, the cells may reach $70-80 \%$ confluency during the selection period, at which point the cultures need to be split. Because the timing of the splitting cannot be predicted in advance, it will not be mentioned in the protocol. However, instead of refreshing the E8 medium supplemented with the mentioned concentrations of puromycin and G418, one can split the hiPSC culture as a normal hiPSC culture (including plating the cells on vitronectincoated plates). The only exception is that the E8 medium should be supplemented with the mentioned concentrations of the antibiotics to continue the selection.

1. Warm $12 \mathrm{~mL}$ E8 medium with $1 \%(\mathrm{v} / \mathrm{v})$ penicillin/streptomycin to room temperature. Add puromycin and G418 for selection; different amounts of the antibiotics are added during the selection period (Table 1).

2. Estimate the efficiency of the transduction by estimating the percentage of G418- and puromycin-resistant cells. To estimate the percentage of resistant cells, estimate the percentage of dead cells (nonresistant cells) for the different conditions (the cultures transduced with the different amounts of lentivirus suspension) and for the nontransduced cells (the cells that serve as a selection control). Calculate the percentage of resistant cells as [100\% - (percentage of dead cells)].

NOTE: If the transductions with the different amounts of lentivirus suspension were performed in duplicate, the transduction efficiency can be estimated more accurately. The condition with the non-transduced cells serves as a selection control; the percentages of dead cells for the cultures transduced with the different amounts of lentivirus suspension should be lower. The estimated percentage of resistant cells is used to choose the hiPSCs that are likely positive for both transgenes. In general, we choose the hiPSCs from the transduction condition were $>90 \%$ of the cells survive the $5 \mathrm{~d}$ selection period.

3. Aspirate the spent medium of the hiPSCs and add $1 \mathrm{~mL}$ of the prepared E8 medium to the wells. Place the 12 well plate $\mathrm{O} / \mathrm{N}$ in a humidified $37^{\circ} \mathrm{C}$ incubator with an atmosphere of $5 \% \mathrm{CO}_{2}$.

\begin{tabular}{|l|l|l|}
\hline & Final concentration of G418 & Final concentration of puromycin \\
\hline Day 4 & $250 \mu \mathrm{g} / \mathrm{mL}$ & $2 \mu \mathrm{g} / \mathrm{mL}$ \\
\hline Day 5 & $250 \mu \mathrm{g} / \mathrm{mL}$ & $2 \mu \mathrm{g} / \mathrm{mL}$ \\
\hline Day 6 & $250 \mu \mathrm{g} / \mathrm{mL}$ & $1 \mu \mathrm{g} / \mathrm{mL}$ \\
\hline Day 7 & $250 \mu \mathrm{g} / \mathrm{mL}$ & $1 \mu \mathrm{g} / \mathrm{mL}$ \\
\hline Day 8 & $250 \mu \mathrm{g} / \mathrm{mL}$ & $1 \mu \mathrm{g} / \mathrm{mL}$ \\
\hline
\end{tabular}

Table 1: Concentrations of Antibiotics during the Selection Period. Concentrations of the puromycin and G418 during the $5 \mathrm{~d}$ of the selection period.

5. Stop the selection and start regular culturing (day 9 and later)

1. After the $5 \mathrm{~d}$ selection period, culture the $r t T A / N g n 2-p o s i t i v e ~ h i P S C s$ as normal hiPSCs, with the exception that the E8 medium of the cells is supplemented with $\mathrm{G} 418$ to a final concentration of $50 \mu \mathrm{g} / \mathrm{mL}$ and with puromycin to a final concentration of $0.5 \mu \mathrm{g} / \mathrm{mL}$. NOTE: The cells can now be frozen (according to standard protocols for cryopreservation of cells) to serve as a backup. This is an important step for the reproducibility of the differentiation protocol, because it allows the use of the same batch of $r$ TA/Ngn2-positive hiPSCs for many future differentiation experiments. 


\section{Differentiation of rtTA/Ngn2-positive hiPSCs to Neurons on 6-well MEAs and Glass Coverslips}

NOTE: In this protocol, the details are provided for differentiating rtTA/Ngn2-positive hiPSCs on two different substrates, i.e. 6-well MEAs (devices composed of 6 independent wells with 9 recording and 1 reference embedded microelectrodes per well) and glass coverslips in the wells of a 24-well plate. The protocols, however, can easily be adapted for larger substrates (e.g., for the wells of 12- or 6-well plates), by scaling up the mentioned values according to the surface area.

1. Prepare the MEAs or glass coverslips (day 0 and day 1 )

1. The day before the start of the differentiation, sterilize the MEAs according to the manufacturer's recommendation.

2. Dilute the adhesion protein Poly-L-Ornithine (PLO) in sterile ultrapure water to a final concentration $50 \mu \mathrm{g} / \mathrm{mL}$. Coat the active electrode area of 6-well MEAs by placing a $100 \mu \mathrm{L}$ drop of the diluted PLO in each well. Place the coverslips in the wells of the 24-well plate using sterile tweezers. Add $800 \mu \mathrm{L}$ of the diluted PLO in each well. Prevent the coverslips from floating by pushing them down with the $1000 \mu \mathrm{L}$ pipette tip.

3. Incubate the 6-well MEAs and 24-well plate $\mathrm{O} / \mathrm{N}$ in a humidified $37^{\circ} \mathrm{C}$ incubator with an atmosphere of $5 \% \mathrm{CO}_{2}$. The next day, aspirate the diluted PLO. Wash the glass surfaces of the 6-well MEAs and the coverslips twice with sterile ultrapure water.

4. Dilute laminin in cold DMEM/F12 to a final concentration of $20 \mu \mathrm{g} / \mathrm{mL}$ (for the 6-well MEAs) and $10 \mu \mathrm{g} / \mathrm{mL}$ (for the glass coverslips). Immediately coat the active electrode area of the 6 -well MEAs by placing a $100 \mu \mathrm{L}$ drop in each well. Similarly, add $400 \mu \mathrm{L}$ of the diluted laminin in each well of the 24-well plate to coat the coverslips. Prevent the coverslips from floating by pushing them down with the $1,000 \mu \mathrm{L}$ pipette tip.

5. Incubate the 6-well MEAs and 24 well plate in a humidified $37{ }^{\circ} \mathrm{C}$ incubator with an atmosphere of $5 \% \mathrm{CO}_{2}$ for at least $2 \mathrm{~h}$.

2. Plate the hiPSCs (day 1)

NOTE: The volumes that are mentioned in steps 3.2.1 - 3.2.4 assume that the rtTA/Ngn2-positive hiPSCs are cultured in a 6-well plate and that the cells of one well are harvested. The volumes that are required for plating the cells on the 6-well MEAs and/or the coverslips depends on the number of 6-well MEAs and/or the number of coverslips that are used in the experiment; the numbers specified in steps 3.2.6 - 3.2.8 allow scaling to different experiment sizes.

1. Warm DMEM/F12, CDS and E8 medium with $1 \%(\mathrm{v} / \mathrm{v})$ penicillin/streptomycin to R/T. Add doxycycline to a final concentration of $4 \mu \mathrm{g} /$ $\mathrm{mL}$ and ROCK inhibitor to the E8 medium.

2. Aspirate the spent medium of the rtTA/Ngn2-positive hiPSCs and add $1 \mathrm{~mL}$ CDS to the hiPSCs. Incubate $3-5$ min in a humidified 37 ${ }^{\circ} \mathrm{C}$ incubator with an atmosphere of $5 \% \mathrm{CO}_{2}$. Check under the microscope whether the cells are detaching from one another.

3. Add $2 \mathrm{~mL} \mathrm{DMEM/F12} \mathrm{in} \mathrm{the} \mathrm{well,} \mathrm{gently} \mathrm{suspend} \mathrm{the} \mathrm{cells} \mathrm{with} \mathrm{a} 1,000 \mu \mathrm{L}$ pipette and transfer the cells to a $15 \mathrm{~mL}$ tube. Add $7 \mathrm{~mL}$ $\mathrm{DMEM} / \mathrm{F} 12$ to the cell suspension. Spin the cells at $200 \mathrm{xg}$ for $5 \mathrm{~min}$.

4. Aspirate the supernatant and add $2 \mathrm{~mL}$ of the prepared E8 medium. Dissociate the hiPSCs by putting the tip of a $1,000 \mu \mathrm{L}$ pipette against the side of the $15 \mathrm{~mL}$ tube and resuspending the cells gently. Check under the microscope whether the cells are dissociated.

5. Determine the number of cells (cells $/ \mathrm{mL}$ ) using a hemocytometer chamber.

NOTE: A 6-well plate well at $80-90 \%$ confluency will typically yield $3.0-4.0 \times 10^{6}$ cells in total.

6. Aspirate the diluted laminin. For the 6-well MEAs, dilute the cells to obtain a cell suspension of $7.5 \times 10^{5}$ cells $/ \mathrm{mL}$. Plate the cells by adding a drop of $100 \mu \mathrm{L}$ of the cell suspension on the active electrode area in each well of the 6-well MEAs. For the coverslips, dilute the cells to obtain a cell suspension of $4.0 \times 10^{4}$ cells $/ \mathrm{mL}$. Plate the cells by adding $500 \mu \mathrm{L}$ of the cell suspension to the wells of the $24-$ well plate.

NOTE: The final cell density on the MEAs is higher than on the coverslips (Figure 1A and B). We found that this high cell density was required for proper recording of the network activity. In the protocol, the numbers are provided that turned out to be optimal for the assays.

7. Place the 6-well MEAs and 24 well plate in a humidified $37^{\circ} \mathrm{C}$ incubator with an atmosphere of $5 \% \mathrm{CO}_{2}$ for $2 \mathrm{~h}(\mathrm{MEAs})$ or $\mathrm{O} / \mathrm{N}(24$ well plate).

8. After $2 \mathrm{~h}$, carefully add $500 \mu \mathrm{L}$ of the prepared E8 medium to each well of the 6-well MEAs. Place the 6-well MEAs O/N in a humidified $37^{\circ} \mathrm{C}$ incubator with an atmosphere of $5 \% \mathrm{CO}_{2}$.

3. Change the medium (day 2)

1. The next day, prepare DMEM/F12 with $1 \%(\mathrm{v} / \mathrm{v}) \mathrm{N}-2$ supplement, $1 \%(\mathrm{v} / \mathrm{v})$ non-essential amino acids and $1 \%(\mathrm{v} / \mathrm{v}) \mathrm{penicillin} /$ streptomycin. Add human recombinant neurotrophin-3 (NT-3) to a final concentration of $10 \mathrm{ng} / \mathrm{mL}$, human recombinant brain-derived neurotrophic factor (BDNF) to a final concentration of $10 \mathrm{ng} / \mathrm{mL}$, and doxycycline to a final concentration of $4 \mu \mathrm{g} / \mathrm{mL}$. Warm the medium to $37^{\circ} \mathrm{C}$.

2. Add laminin to a final concentration of $0.2 \mu \mathrm{g} / \mathrm{mL}$ to the medium. Filter the resulting medium. Aspirate the spent medium from the wells of the 6-well MEAs and the 24-well plate and replace it with the prepared medium. Incubate the 6-well MEAs and 24-well plate O/N in a humidified $37{ }^{\circ} \mathrm{C}$ incubator with an atmosphere of $5 \% \mathrm{CO}_{2}$.

4. Add rat astrocytes (day 3)

NOTE: The volumes that are mentioned in this protocol assume that the rat astrocytes are cultured in T75 culture flasks. It is critical that the rat astrocytes that are added to the cultures are of good quality. We use two criteria to check if the rat astrocytes are of good quality. First, the rat astrocyte culture should be able to grow confluent within ten days after the isolation from the rat embryonic brains. Second, after splitting the rat astrocyte culture, the rat astrocytes should be able to form a confluent, tessellated monolayer (Figure 1C). If the rat astrocyte culture does not fulfill these two criteria, we advise not to use this culture for differentiation experiments.

1. Warm $0.05 \%$ trypsin-EDTA to RT. Warm the DPBS and DMEM/F12 with $1 \%(\mathrm{v} / \mathrm{v})$ penicillin/streptomycin to $37^{\circ} \mathrm{C}$.

2. Aspirate the spent medium of the rat astrocyte culture. Wash the culture by adding $5 \mathrm{~mL} \mathrm{DPBS}$ and swish it around gently.

3. Aspirate the DPBS and add $5 \mathrm{~mL} 0.05 \%$ trypsin-EDTA. Swish the trypsin-EDTA around gently. Incubate in a humidified $37{ }^{\circ} \mathrm{C}$ incubator with an atmosphere of $5 \% \mathrm{CO}_{2}$ for $5-10 \mathrm{~min}$.

4. Check under the microscope whether the cells are detached. Detach the last cells by hitting the flask a few times. 
5. Add $5 \mathrm{~mL}$ of DMEM/F12 to the flask. Triturate the cells gently inside the flask with a $10 \mathrm{~mL}$ pipette. Collect the cell suspension in a 15 $\mathrm{mL}$ tube. Spin the tube at $200 \mathrm{xg}$ for $8 \mathrm{~min}$.

6. Aspirate the supernatant and resuspend the cells in $1 \mathrm{~mL}$ of DMEM/F12. Determine the number of cells (cells $/ \mathrm{mL}) \mathrm{using}$ a hemocytometer chamber.

7. Add $7.5 \times 10^{4}$ astrocytes per well of the 6-well MEAs. Add $2.0 \times 10^{4}$ astrocytes per well of the 24 -well plate. Incubate the MEAs and the 24-well plate $\mathrm{O} / \mathrm{N}$ in a humidified $37{ }^{\circ} \mathrm{C}$ incubator with an atmosphere of $5 \% \mathrm{CO}_{2}$.

\section{Change the medium (day 4 )}

1. Prepare neurobasal medium with $2 \%(\mathrm{v} / \mathrm{v}) \mathrm{B}-27$ supplement, $1 \%(\mathrm{v} / \mathrm{v}) \mathrm{L}$-alanyl-L-glutamine and $1 \%(\mathrm{v} / \mathrm{v})$ penicillin/streptomycin. Add NT-3 to a final concentration of $10 \mathrm{ng} / \mathrm{mL}$, BDNF to a final concentration of $10 \mathrm{ng} / \mathrm{mL}$, and doxycycline to a final concentration of $4 \mu \mathrm{g} /$ $\mathrm{mL}$. In addition, add cytosine $\beta$-D-arabinofuranoside to a concentration of $2 \mu \mathrm{M}$.

NOTE: Cytosine $\beta$-D-arabinofuranoside is added to the medium to inhibit astrocyte proliferation and to kill the remaining hiPSCs that are not differentiating into neurons.

2. Filter the medium and warm to $37^{\circ} \mathrm{C}$. Aspirate the spent medium from the wells of the 6-well MEAs and the 24-well plate and replace it with the prepared medium. Maintain the 6 -well MEAs and the 24 -well plate in a humidified $37^{\circ} \mathrm{C}$ incubator with an atmosphere of $5 \%$ $\mathrm{CO}_{2}$.

6. Refresh the medium (day 6 - 28)

NOTE: Starting from day 6, refresh half of the medium every two days. From day 10 onwards, the medium is supplemented with FBS to support the astrocyte viability.

1. Prepare neurobasal medium with $2 \%(\mathrm{v} / \mathrm{v}) \mathrm{B}-27$ supplement, $1 \%(\mathrm{v} / \mathrm{v})$ L-alanyl-L-glutamine and $1 \%(\mathrm{v} / \mathrm{v})$ penicillin/streptomycin. Add NT-3 to a final concentration of $10 \mathrm{ng} / \mathrm{mL}$, BDNF to a final concentration of $10 \mathrm{ng} / \mathrm{mL}$, and doxycycline to a final concentration of $4 \mu \mathrm{g} /$ $\mathrm{mL}$. From day 10 onwards, also supplement the medium with $2.5 \%(\mathrm{v} / \mathrm{v}) \mathrm{FBS}$. Filter the resulting medium and warm to $37^{\circ} \mathrm{C}$.

2. Remove half of the spent medium from the wells of the 6-well MEAs and the 24-well plate using a $1000 \mu \mathrm{L}$ pipette and replace it with the prepared medium. Maintain the 6-well MEAs and the 24 -well plate in a humidified $37^{\circ} \mathrm{C}$ incubator with an atmosphere of $5 \% \mathrm{CO}_{2}$.

\section{Establish the Neurophysiological Profile of hiPSC-derived Neurons}

NOTE: Two to three weeks after the induction of differentiation, the hiPSC-derived neurons can be used for different downstream analyses. In this section, examples of some downstream analyses are given that can be performed to establish the neurophysiological profile of the hiPSC-derived neurons.

1. Characterize the neuronal network activity using MEAs

1. Record 20 min of electrophysiological activity of hiPSC-derived neurons cultured on MEAs. During the recording, maintain the temperature at $37{ }^{\circ} \mathrm{C}$, and prevent evaporation and $\mathrm{pH}$ changes of the medium by inflating a constant, slow flow of humidified gas $(5 \%$ $\mathrm{CO}_{2}, 20 \% \mathrm{O}_{2}, 75 \% \mathrm{~N}_{2}$ ) onto the MEA.

2. After 1200X amplification (MEA 1060, MCS), sample the signal at $10 \mathrm{kHz}$ using the MCS data acquisition card. Analyze the data (spike and burst detection) using a custom software package ${ }^{23}$.

2. Characterize the single-cell electrophysiological activity

1. Transfer the cover slips containing the hiPSC-derived neuronal cultures to a submerged fixed-stage recording chamber in an upright microscope. Record 20 min of spontaneous action potential-evoked postsynaptic currents (sEPSC) ${ }^{24}$. Detect the synaptic event using neuroscientific program.

3. Characterize the neuronal morphology and synapsin expression

1. Fix and stain the hiPSC-derived neurons for MAP2, synapsin-1/2, and PSD-95 $22,24,25$. Quantify the number of synapsin-1/2 and PSD-95 puncta using image analysis software.

\section{Representative Results}

Here we have successfully modified a protocol in which hiPSCs are differentiated directly into cortical neurons by over-expressing the transcription factor neurogenin- $2^{12}$ and we have adapted it for the use of MEAs. This approach is fast and efficient allowing us to obtain functional neurons and network activity already during the third week after the induction of differentiation.

During the course of the differentiation protocol, the cells morphologically started to resemble neurons: small processes were formed and neurons started connecting to each other (Figure 1A). We established a neurophysiological profile of the neurons derived from a healthy-control hiPSC line, by measuring their neuronal morphology and synaptic properties during development. hiPSC-derived neurons were stained for MAP2 and synapsin-1/2 at different days after the start of differentiation (Figure 2A). The derived neurons show mature neuronal morphology already 3 weeks after the induction of differentiation. The number of synapsin-1/2 puncta (a measure for the number of synapses) was quantified based on synapsin-1/2 immunocytochemistry stainings. The number of synapsin-1/2 puncta increased over time, suggesting that the level of neuronal connectivity is also increasing (Figure 2B). The number of synapsin-1/2 puncta 23 days after the induction of differentiation was similar in two independent IPS lines (Figure 2C). At 23 DIV most synapsin1/2 puncta were juxtaposed to PSD-95 puncta, which is indicative for functional synapses (Figure 2D).

Consistent with the results described by Zhang et al., we generated a population of excitatory upper layer cortical neurons, confirmed by panneuronal and subtype-specific cortical markers such as BRN2 and SATB2(layer II/III). We did not observe neurons that were positive for deep layer neurons CTIP2 (layer V) or Foxp2 (layer VI) (Figure 2E and F) 
To characterize the electrophysiological activity of the hiPSC-derived neurons, we used whole-cell current and voltage clamp recordings, i.e. intrinsic properties and excitatory input onto these neurons were measured during development. The neurons were able to generate action potentials already one week after the of differentiation and the percentage of spiking cells was increasing over time (Figure $\mathbf{2 G}$ and $\mathbf{H}$ ). Furthermore, the neurons received excitatory synaptic input already a week after the induction of differentiation: both frequency and amplitude of the excitatory synaptic input increased during development (Figure $\mathbf{2} \mathbf{l}-\mathbf{K}$ ).

To better understand how single-cell activity combines to form network-level functions, it is essential to study how neurons work in concert. In vitro neuronal networks cultured on MEAs constitute a valuable experimental model for studying the neuronal dynamics. We recorded 20 min of electrophysiological network activity of neurons derived from a healthy-control hiPSC line cultured on 6-well MEAs (Figure 2M). Few weeks after the induction of differentiation, the neurons derived from healthy-control hiPSCs formed functionally active neuronal networks, showing spontaneous events $(0.62 \pm 0.05$ spike/s; Figure $2 \mathrm{~N})$. At this stage of development (i.e. $16 \mathrm{~d}$ after the start of the differentiation) no synchronous events involving all the channels of the MEAs are detected (Figure 20). The level of network activity increased during the development: during the fourth week after the induction of differentiation, the neuronal networks showed high level of spontaneous activity $(2.5 \pm 0.1$ spike/s; Figure $2 \mathrm{~N})$ in all the wells of the device. The networks also exhibited synchronous network bursts $(4.1 \pm 0.1$ burst/min, Figure 20$)$ with long duration $(2,100 \pm 500 \mathrm{~ms})$.

\section{A}
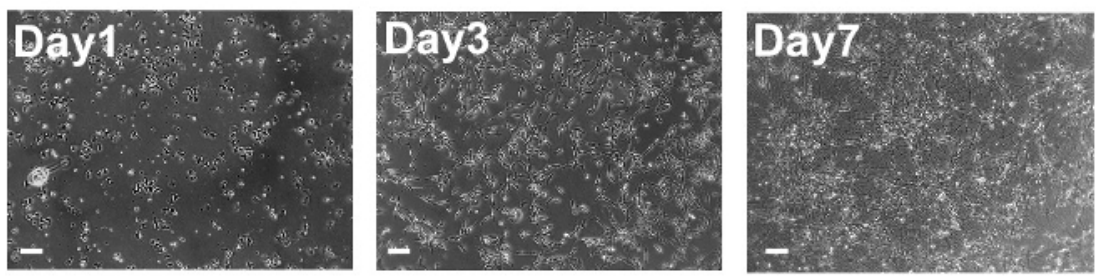

B
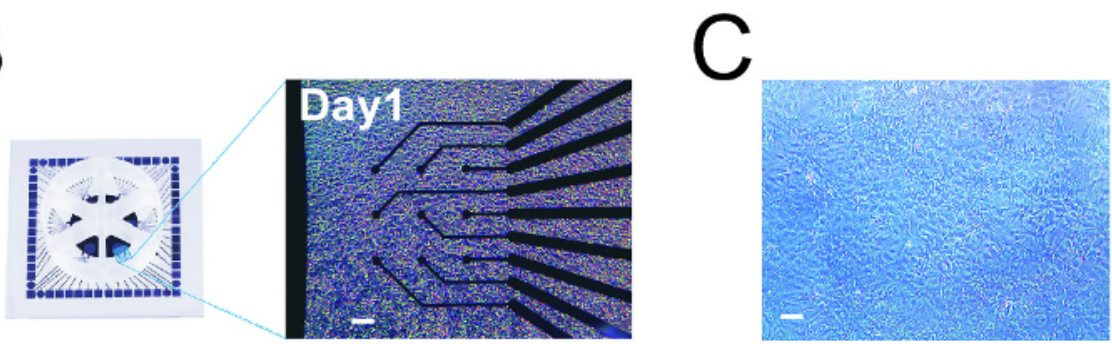

Figure 1: hiPSC Differentiation into Neurons. A. Three time points of hiPSCs differentiation into neurons on coverslips. B. Plating of hiPSCs on MEAs. C. Astrocytes at $100 \%$ confluency in T75 flask (note that the cells form a tessellated monolayer). Scale bars: $150 \mu \mathrm{m}$. Please click here to view a larger version of this figure. 
A

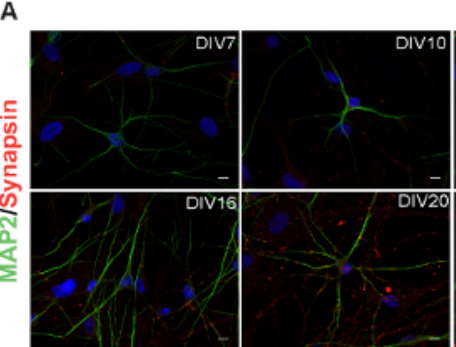

E
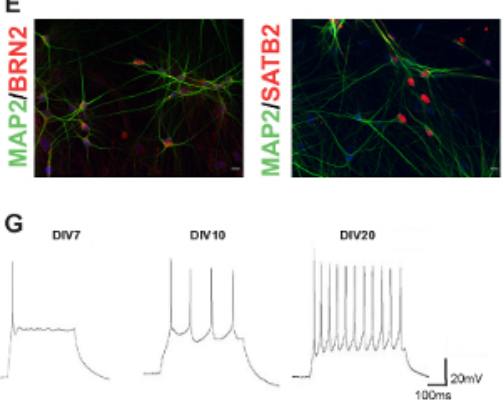

B

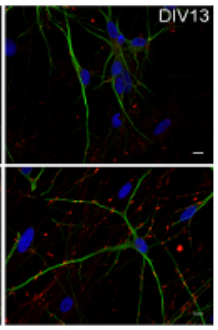

D
C
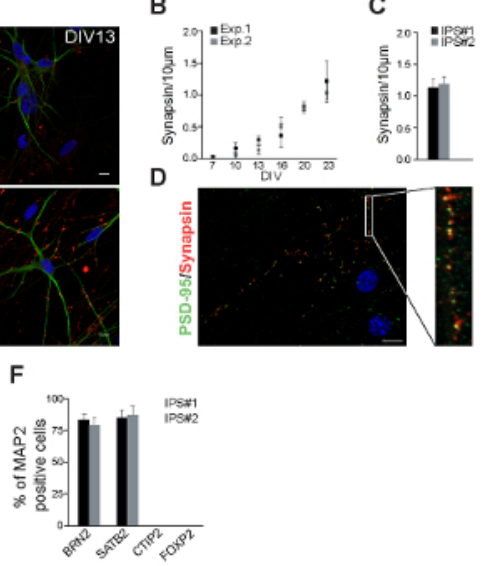

H

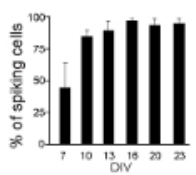

I

on?

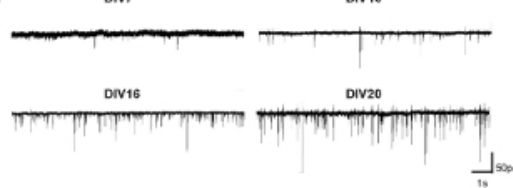

M

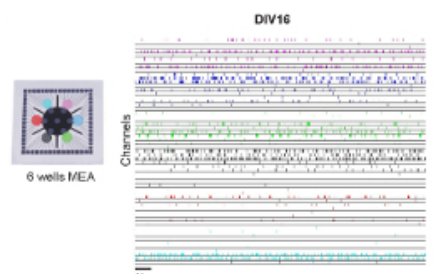

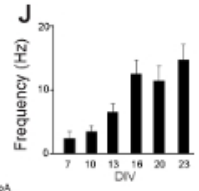

$\sqrt{18}$

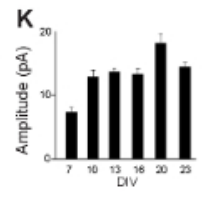

N

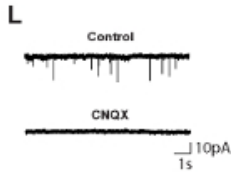

0
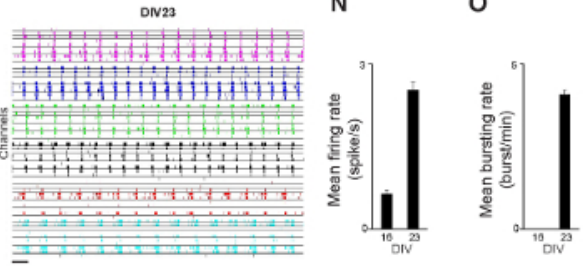

Figure 2. hiPSC-derived Neurons Characterization. A. hiPSC-derived neurons were stained for MAP2 (green) and synapsin-1/2 (red) at different days after the start of differentiation. Scale bar: $10 \mu \mathrm{m}$. B. Quantification of synapsin puncta in two independent experiments. In each experiments at least 10 cells were analyzed. C. Quantification of synapsin puncta at DIV23 in neurons derived from two independent IPS lines. D. hiPSC-derived neurons were stained for PSD-95 (green) and synapsin-1/2 (red) 23 days after the start of differentiation. Synapsin puncta are juxtaposed to PSD-95 puncta. E. hiPSC-derived neurons were stained for MAP2 (green) and BRN2 (red) or SATB2 (red) $23 \mathrm{~d}$ after the start of differentiation. F. Percentage of MAP2 positive cells that were positive for indicated markers. G. Representative current clamp recordings showing that action potentials can be generated as early as $7 \mathrm{~d}$ after the start of differentiation. $\mathbf{H}$. Percentage of cells at different days after the induction of differentiation that show one or more action potentials. I. Representative traces of excitatory postsynaptic currents (EPSCs) received by hiPSC-derived neurons at different days after differentiation. J. Frequency of excitatory postsynaptic currents during development. K. Amplitude of excitatory postsynaptic currents during development. L. Representative traces of EPSC recordings without (control) and with CNQX (CNQX). M. Neurons derived from one hiPSC line were cultured on a 6-well MEA and network activity is shown for hiPSC-derived neuronal networks 16 and $23 \mathrm{~d}$ after the induction of differentiation. The activity recorded from each well (sampling rate of $10 \mathrm{kHz}$ ) is indicated with a different color ( $5 \mathrm{~min}$ of the $20 \mathrm{~min}$ of recording are shown). N. Firing rate 16 and $23 \mathrm{~d}$ after the induction of differentiation. $\mathbf{O}$. Bursting rate 16 and $23 \mathrm{~d}$ after the induction of differentiation. Please click here to view a larger version of this figure.

Given the results, the quality of the resulting hiPSC-derived neurons can be assessed by making a neurophysiological profile of the cells. That is, three to four weeks after the start of the differentiation, the morphology, synapsin-1/2 expression and electrophysiology of the neurons can be assessed. At that time point, the hiPSC-derived neurons are expected to show a neuronal-like morphology, to be MAP2, synapsin/PSD-95 positive when performing immunocytochemistry, and to exhibit spontaneous electrophysiological activity (both at the single-cell and network level).

\section{Discussion}

Here we have implemented an efficient hiPSC-differentiation protocol published by Zhang et al. (2013) ${ }^{12}$ for measuring the network activity of hiPSC-derived neuronal networks on MEAs. We adapted the original protocol by creating an rtTA/Ngn2-positive hiPSC line before inducing neuronal differentiation. This additional step allows us to control the neuronal cell density on the MEA. Control over the neuronal density was an important pre-requisite for adapting the protocol to MEAs and for ensuring consistency. To measure the activity of neuronal networks using MEAs, the neurons need to form dense networks directly on top of the MEA electrodes ${ }^{17,18}$. This necessarily requires tight control over the plating density of the neurons. The rtTA/Ngn2-positive hiPSC line allows for control of neuron density because this tactic does not rely on acute 
lentiviral transductions of hiPSCs prior to differentiation; the rtTA/Ngn2-positive hiPSC line therefore nearly eliminates any variation in the final yield due to, for example, lentiviral toxicity and variable infection efficiency.

Another critical step of the experimental procedure is the number of the rat astrocytes that are cocultured with the differentiating hiPSCs. Astrocytes actively contribute to the refinement of developing neural circuits by controlling synapse formation, maintenance, and elimination, all of which are important processes for neuronal functioning. The protocol presented in this paper is highly astrocyte-dependent: to fully mature and form functional synapses, the neurons require support from the astrocytes. We experienced that the number of astrocytes should be roughly equal to the number of hiPSC-derived neurons to support the maturation of the neurons and the formation of neuronal networks exhibiting spontaneous activity. Since our astrocyte protocol yields primary cell cultures with a limited life span, the isolation of rat astrocytes has to be performed regularly.

Our adaptation of the protocol published by Zhang et al. $(2013)^{12}$ for use with MEA technology will likely significantly improve our ability to study the network activity of hiPSC-derived networks. Previously, protocols used for studying hiPSC-derived neuronal networks with MEAs relied on time-consuming differentiation procedures ${ }^{13-16}$. The protocol from Zhang et al. (2013) provides a rapid alternative, and our modification removes a source of variability, which makes it now more feasible to use hiPSC-derived neurons in combination with MEA technology, especially in highthroughput or pharmacological studies. In addition, because the method published by Zhang et al. (2013) ${ }^{12}$ yields a homogeneous population of upper-layer cortical neurons, our adapted protocol makes possible focused studies into the network activity of this particular neuronal subset.

Nonetheless, this approach has also several limitations. First, the homogeneity of the cultures can also be considered a disadvantage, because the cultures are less likely to resemble in vivo networks, where different classes of neurons (i.e. inhibitory and excitatory neurons) constitute a heterogeneous network. To further enhance the use of the hiPSC-derived neurons with MEA technology, it will be important to develop rapid (transgene-based) differentiation protocols for other neuronal cell populations. If protocols become available, the in vitro networks would mimic in vivo networks more closely. Second, at present rat astrocytes must be added to the hiPSC-derived neurons for growth support, and therefore the resulting neuronal network is not a human neuronal network sensu stricto. Reliable protocols for differentiating hiPSCs into astrocytes may in the future solve this problem ${ }^{26}$. Third, two-dimensional neuronal networks, as described here, are a limited model for studying complex threedimensional in vivo neuronal networks. Fortunately, protocols describing three-dimensional cultures of rat primary neurons in combination with MEA technology are already available ${ }^{27,28}$. Prospectively, the combination of rapid differentiation protocols for obtaining hiPSC-derived neurons and astrocytes with three-dimensional culture techniques and MEA technology should provide novel insight into the biological mechanisms underlying neurological disorders.

\section{Disclosures}

The authors have nothing to disclose.

\section{Acknowledgements}

The authors thank Jessica Classen for performing the whole-cell patch-clamp experiments. The hiPSCs used in our experiments were kindly provided by Huiqing Zhou and Willem van den Akker from the Radboud University Nijmegen. The transfer vectors used in this protocol were kindly provided by Oliver Brüstle, Philipp Koch and Julia Ladewig from the University of Bonn Medical Centre.

\section{References}

1. Ahfeldt, T., Litterman, N.K., \& Rubin, L. Studying human disease using human neurons. Brain Res. (2016).

2. Zhang, S. C., Wernig, M., Duncan, I. D., Brustle, O., \& Thomson, J. A. In vitro differentiation of transplantable neural precursors from human embryonic stem cells. Nat Biotechnol. 19 (12), 1129-1133 (2001).

3. Perrier, A. L. et al. Derivation of midbrain dopamine neurons from human embryonic stem cells. Proc Natl Acad Sci USA. 101 (34), 12543-12548 (2004).

4. $\mathrm{Wu}, \mathrm{H}$. et al. Integrative genomic and functional analyses reveal neuronal subtype differentiation bias in human embryonic stem cell lines. Proc Natl Acad Sci USA. 104 (34), 13821-13826 (2007).

5. Chambers, S. M., Fasano, C. A., Papapetrou, E. P., Tomishima, M., Sadelain, M., \& Studer, L. Highly efficient neural conversion of human ES and iPS cells by dual inhibition of SMAD signaling. Nat Biotechnol. 27 (3), 275-280 (2009).

6. Shi, Y., Kirwan, P., \& Livesey, F.J. Directed differentiation of human pluripotent stem cells to cerebral cortex neurons and neural networks. Nat Protoc. 7, 1836-1846 (2012).

7. Espuny-Camacho, I. et al. Pyramidal neurons derived from human pluripotent stem cells integrate efficiently into mouse brain circuits in vivo. Neuron. 77 (3), 440-456 (2013).

8. Maroof, A. M. et al. Directed differentiation and functional maturation of cortical interneurons from human embryonic stem cells. Cell Stem Cell. 12 (5), 559-572 (2013).

9. Johnson, M. A., Weick, J. P., Pearce, R. A., \& Zhang, S. C. Functional neural development from human embryonic stem cells: accelerated synaptic activity via astrocyte coculture. J Neurosci. 27 (12), 3069-3077 (2007).

10. Hu, B. Y. et al. Neural differentiation of human induced pluripotent stem cells follows developmental principles but with variable potency. Proc Natl Acad Sci USA. 107 (9), 4335-4340 (2010).

11. Kim, H. et al. miR-371-3 expression predicts neural differentiation propensity in human pluripotent stem cells. Cell Stem Cell. 8 (6), $695-706$ (2011).

12. Zhang, Y. et al. Rapid single-step induction of functional neurons from human pluripotent stem cells. Neuron. 78 (5), $785-798$ (2013).

13. Odawara, A., Saitoh, Y., Alhebshi, A. H., Gotoh, M., \& Suzuki, I. Long-term electrophysiological activity and pharmacological response of a human induced pluripotent stem cell-derived neuron and astrocyte co-culture. Biochem Biophys Res Commun. 443 (4), 1176-1181 (2014) 
14. Odawara, A., Katoh, H., Matsuda, N., \& Suzuki, I. Physiological maturation and drug responses of human induced pluripotent stem cellderived cortical neuronal networks in long-term culture. Sci Rep. 6, 26181 (2016).

15. Amin, H., Maccione, A., Marinaro, F., Zordan, S., Nieus, T., \& Berdondini, L. Electrical Responses and Spontaneous Activity of Human iPSDerived Neuronal Networks Characterized for 3-month Culture with 4096-Electrode Arrays. Front Neurosci. 10 (2016).

16. Heikkila, T. J. et al. Human embryonic stem cell-derived neuronal cells form spontaneously active neuronal networks in vitro. Exp Neurol. 218 (1), 109-116 (2009).

17. Massobrio, P., Massobrio, G., \& Martinoia, S. Multi-program approach for simulating recorded extracellular signals generated by neurons coupled to microelectrode arrays. Neurocomputing. 70, 2467-2476 (2007).

18. Wang, L., Riss, M., Buitrago, J.O., \& Claverol-Tinturé, E. Biophysics of microchannel-enabled neuron-electrode interfaces. J Neural Eng. 9 (2) (2012).

19. McCarthy, K. D., \& de Vellis, J. Preparation of separate astroglial and oligodendroglial cell cultures from rat cerebral tissue. J Cell Biol. 85 , 890-902 (1980).

20. Schildge, S., Bohrer, C., Beck, K., \& Schachtrup, C. Isolation and culture of mouse cortical astrocytes. J. Vis. Exp. (71), e50079 (2013).

21. Pacifici, M., \& Peruzzi, F. Isolation and culture of rat embryonic neural cells: a quick protocol. J Vis Exp. (63), e3965 (2012).

22. Ba, W., et al. ARHGAP12 functions as a developmental brake on excitatory synapse function. Cell Rep. 14 (6), 1355-1368 (2016).

23. Bologna, L.L. et al. Investigating neuronal activity by SPYCODE multi-channel data analyzer. Neural Netw. 23 (6), 685-697 (2010).

24. Ba, W. et al. TRIO loss of function is associated with mild intellectual disability and affects dendritic branching and synapse function. Hum Mol Genet. 25 (5), 892-902 (2016).

25. Benevento, M. et al. Histone methylation by the Kleefstra syndrome protein EHMT1 mediates homeostatic synaptic scaling. Neuron. 91 (2), 341-355 (2016).

26. Krencik, R., Weick, J. P., Liu, Y., Zhang, Z.-J., \& Zhang, S.-C. Specification of transplantable astroglial subtypes from human pluripotent stem cells. Nature biotechnology. 29 (6), 528-534 (2011).

27. Frega, M., Tedesco, M., Massobrio, P., Pesce, M., \& Martinoia, S. Network dynamics of 3D engineered neuronal cultures: a new experimental model for in-vitro electrophysiology. Sci Rep. 4, 5489 (2014).

28. Tedesco, M., Frega, M., Martinoia, S., Pesce, M., \& Massobrio, P. Interfacing 3D engineered neuronal cultures to micro-electrode arrays: an innovative in vitro experimental model. $J$ Vis Exp. (104), e53080 (2015). 